\title{
Exploring the chemical composition, emerging applications, potential uses, and health benefits of durian: a review
}

\begin{abstract}
Durian (Durio zibethinus) is an exotic tropical fruit grown in Malaysia and throughout the Southeast Asian region. The expansion of the durian industry and the advancement in processing of durian products has significantly expanded due to international trade. As an exotic fruit, durian is relished for its strong aroma and unique taste. Durian has been recognized as an important fruit especially for the underlying nutritional attributes of the fruit. Apart from the nutritional value of fruit, the presence of volatile organic compounds as well as phytochemical properties is also discussed. Durians are also a rich source of volatile compounds that can benefit human health and the food industries. This review focuses on the chemical composition, potential uses, health benefits, and emerging technologies for the quality evaluation of durian, with the goal of providing information for its exploitation. The innovation of non-destructive techniques to evaluate the quality of durian can be implemented to produce better yield and to improve the existing applications. Furthermore, the challenges and future perspectives regarding the potential uses of the fruit are proposed.
\end{abstract}

Keyword: Durian; Chemical composition; Nutritional value; Health benefits; Exotic fruit 